\title{
Icariin inhibits oral squamous cell carcinoma cell proliferation and induces apoptosis via inhibiting the NF-kB and PI3K/AKT pathways
}

\author{
LING SUN $^{1}$ and JING ZHANG ${ }^{2}$ \\ ${ }^{1}$ Department of Pharmacy, Jiangsu Vocational College of Medicine, Yancheng, Jiangsu 224005; \\ ${ }^{2}$ Department of Hematology, Yancheng No. 1 People's Hospital, Yancheng, Jiangsu 224006, P.R. China
}

Received November 4, 2020; Accepted March 15, 2021

DOI: $10.3892 /$ etm.2021.10374

\begin{abstract}
Oral squamous cell carcinoma (OSCC), one of the most common types of human cancer, has a high mortality rate and a poor prognosis due to its high rates of recurrence and metastasis. In recent years, icariin (ICA) has been reported to play an important role in a variety of malignancies, such as gastric, colorectal, pancreatic and ovarian cancer. However, its role and mechanism in OSCC remains to be elucidated. The present study aimed to investigate the effect of ICA in OSCC cells and to reveal its underlying mechanisms. The OSCC cell lines SCC9 and Cal 27 were used to explore the effect of different concentrations of ICA on the biological behavior of OSCC cells. The effect of ICA on OSCC cell proliferation and apoptosis was determined using 3-(4,5-dimethylthiazol-2-yl)-2,5-diphenyl-2H-tetrazolium bromide and flow cytometric assays, respectively. Subsequently, the protein expression levels of caspase-3 and cleaved-caspase-3 were detected using western blot analysis. Additionally, the protein and mRNA expression levels of nuclear factor- $\kappa \mathrm{B}(\mathrm{NF}-\kappa \mathrm{B})$ and phosphatidylinositol-3-kinase $(\mathrm{PI} 3 \mathrm{~K}) /$ protein kinase B (AKT) signaling pathway-related factors were determined using western blot analysis and reverse transcription-quantitative PCR, respectively. The results demonstrated that ICA inhibited OSCC cell proliferation and significantly increased the apoptosis rate in a dose-dependent manner. In addition, treatment of OSCC cells with ICA upregulated the protein expression of cleaved-caspase- 3 and increased the cleaved-caspase-3/caspase- 3 ratio. The protein expression levels of phosphorylated (p)-p65, p-PI3K and p-AKT were decreased in OSCC cells treated with ICA. The aforementioned findings revealed that ICA could attenuate the
\end{abstract}

Correspondence to: Professor Ling Sun, Department of Pharmacy, Jiangsu Vocational College of Medicine, 283 Jiefang South Road, Yancheng, Jiangsu 224005, P.R. China

E-mail: sunling1104@163.com

Key words: icariin, oral squamous cell carcinoma, proliferation, apoptosis, NF-кB, PI3K/AKT pathway proliferation of OSCC cells and induce apoptosis via inhibiting the $\mathrm{NF}-\kappa \mathrm{B}$ and PI3K/AKT signaling pathways. Therefore, the current study provided a new insight into the clinical treatment of OSCC.

\section{Introduction}

Icariin (ICA), a flavonoid extract of epimedium, has a wide range of pharmacological effects, such as anti-inflammatory, anti-oxidant and antitumor effects in the cardio-cerebral vascular, nervous and urogenital systems and malignant tumors (1-4). Chinese medicine has been widely used to treat several types of malignant tumors. ICA, as a vital active ingredient of epimedium in Chinese medicine, serves an inhibitory role in the occurrence and development of malignant tumors; therefore, it has been widely used in the prevention and treatment of numerous types of cancer, such as cervical, ovarian, colon and triple-negative breast cancer (5-8).

Oral squamous cell carcinoma (OSCC) occurs in the oral mucosa, and is characterized by strong local invasion and easy metastasis to the cervical lymph nodes $(9,10)$. Furthermore, ICA is considered as a novel biological immune modulator and inducer of differentiation, and it has been reported to improve immune function, inhibit tumor cell proliferation, tumor growth and angiogenesis, induce tumor cell apoptosis and alter tumor cell cycle distribution $(11,12)$. Compared with other traditional antitumor drugs, ICA can regulate tumor immunity and reduce the lethality of cells of the surrounding normal tissues. Li et al (13) demonstrated that ICA could inhibit hepatocellular carcinoma cell proliferation, promote apoptosis and enhance the antitumor effects of arsenic trioxide in vitro and in vivo. Additionally, Yang et al (14) revealed that ICA not only inhibited the proliferation of glioblastoma cells in a dose-dependent manner, but also enhanced the antitumor effect of temozolomide. Shi et al (15) also found that ICA attenuated the proliferation of colorectal cancer (CRC) cells and inhibited the antitumor activity of 5-fluorouracil in CRC via inhibiting the activity of nuclear factor $-\kappa \mathrm{B}(\mathrm{NF}-\kappa \mathrm{B})$ in vitro. The $\mathrm{NF}-\kappa \mathrm{B}$ signaling pathway is closely associated with tumor cell apoptosis and plays a key role in the immune aging process of tumor cells (16-18). The aforementioned studies indicate that ICA has a wide range 
of potential antitumor effects. However, its effects on OSCC remain elusive.

The phosphatidylinositol-3-kinase (PI3K)/protein kinase B (AKT) signaling pathway has been reported to play critical roles in various types of cancer (19-21). For example, the PI3K/AKT pathway is regarded as one of the key mechanisms involved in lung cancer cell metastasis and the epithelial-mesenchymal transition (19). Besides, the PI3K/AKT signaling pathway is associated with renal cell carcinoma cell proliferation and metastasis (20). Moreover, the PI3K/AKT pathway plays important roles in the regulation of human pharyngeal squamous carcinoma cell apoptosis (21). Activation of the PI3K/AKT signaling pathway has been confirmed in OSCC, and inhibiting this signaling pathway has been reported to attenuate the development of OSCC (22-24). Until now, whether ICA could affect PI3K/AKT signaling pathway activation in OSCC remain unclear. Therefore, the present study aimed to investigate the effects and underlying molecular mechanisms of ICA on OSCC cells to provide a novel theoretical basis for the treatment of OSCC.

\section{Materials and methods}

Cell culture and treatment. The OSCC cell lines Cal 27 and SCC9 were obtained from the American Type Culture Collection, and human oral mucosa fibroblasts were purchased from Shanghai Aiyan Biotechnology Co., Ltd. All cells were cultured in Dulbecco's modified Eagle's medium supplemented with $10 \%$ fetal bovine serum (both Gibco; Thermo Fisher Scientific, Inc.), $100 \mathrm{U} / \mathrm{ml}$ penicillin and $100 \mu \mathrm{g} / \mathrm{ml}$ streptomycin at $37^{\circ} \mathrm{C}$ in a humidified incubator containing $5 \% \mathrm{CO}_{2}$. Human normal oral keratinocytes (hNOKs) were obtained from Lifeline ${ }^{\circledast}$ Cell Technology. hNOKs were cultured in DermaLife ${ }^{\circledR}$ K Medium (Lifeline ${ }^{\circledR}$ Cell Technology). Cells were treated with 0, 5, 10, 20 and $40 \mu \mathrm{M}$ ICA (Shanghai Yuanye Biotechnology Co., Ltd.) for 24, 48 and $72 \mathrm{~h}$.

\section{3-(4,5-Dimethylthiazol-2-yl)-2,5-diphenyl-2H-tetrazolium} bromide (MTT) assay. OSCC cell proliferation was evaluated using a MTT assay. Briefly, Cal 27 and SCC 9 cells were seeded $\left(10^{4}\right.$ cells per well) into 96 -well plates and cultured at $37^{\circ} \mathrm{C}$ in a humidified incubator containing 5\% $\mathrm{CO}_{2}$. Subsequently, cells were treated with $0,5,10,20$ and $40 \mu \mathrm{M}$ ICA for 24,48 or $72 \mathrm{~h}$. Following treatment, cells were supplemented with $10 \mu \mathrm{l}$ MTT solution and incubated for an additional $4 \mathrm{~h}$, according to the manufacturer's instructions. The formazan crystals were dissolved by the addition of dimethyl sulfoxide. Finally, optical density values were measured at $570 \mathrm{~nm}$ using a multifunctional plate reader (BD Biosciences).

Flow cytometric analysis. Cell apoptosis was assessed using an Annexin-V/PI Apoptosis Detection kit (Beyotime Institute of Biotechnology). Cal 27 and SCC9 cells were treated with 0, 5, 10, 20 and $40 \mu \mathrm{M}$ ICA for $48 \mathrm{~h}$. Subsequently, cells in the logarithmic phase of growth were digested with $0.25 \%$ trypsin solution without ethylene diamine tetra acetic acid, centrifuged at $1,000 \mathrm{x}$ g for $5 \mathrm{~min}$ at $4^{\circ} \mathrm{C}$ and the supernatant was discarded. The cell pellet was then washed twice with pre-chilled PBS and re-suspended in $100 \mu \mathrm{l}$ of pre-chilled $1 \mathrm{X}$ Annexin V binding buffer (Beyotime Institute of Biotechnology). The cells were then incubated with $5 \mu \mathrm{l}$ Annexin V-FITC and $5 \mu \mathrm{l}$ propidium iodide (Beyotime Institute of Biotechnology) for $15 \mathrm{~min}$ at room temperature in the dark. To detect apoptosis, a BD FACSCalibur flow cytometer (BD Biosciences) was used, and data were analyzed using the Cell Quest software (version 5.1; BD Biosciences).

Western blot analysis. The expression levels of cleaved-caspase-3 (cat. no. ab32042; dilution, 1:500; Cell Signaling Technology, Inc.), pro-caspase-3 (cat. no. ab32150; dilution rate, 1:1,000; Abcam), Bcl-2 (cat. no. 4223; dilution, 1:1,000; Cell Signaling Technology, Inc.), Bax (cat. no. 5023; dilution, 1:1,000), phosphorylated (p)-p65 (cat. no. 3033; dilution, 1:1,000), p65 (cat. no. 8242; dilution, 1:1,000) and p-AKT (cat. no. 4060; dilution, 1:1,000) and AKT (cat. no. 4685; dilution, 1:1,000) (all Cell Signaling Technology, Inc.) were evaluated using western blot analysis. Total proteins were extracted from OSCC cells using RIPA lysis buffer (Beyotime Institute of Biotechnology), and the protein concentration was determined using a bicinchoninic acid assay kit (Sigma-Aldrich; Merck KGaA), according to the manufacturer's protocol. Proteins ( $40 \mu \mathrm{g}$ per lane) were separated by $10 \%$ SDS-PAGE and then transferred onto a polyvinylidene fluoride (PVDF) membrane (EMD Millipore). Following blocking with 5\% skimmed milk for $1 \mathrm{~h}$ at room temperature, the membrane was first incubated with primary antibodies at $4^{\circ} \mathrm{C}$ overnight and then with anti-rabbit IgG horseradish peroxidase-linked antibodies (cat. no. 7074; dilution, 1:2,000; Cell Signaling Technology, Inc.) at room temperature for $1 \mathrm{~h}$. Finally, the blot was developed with enhanced chemiluminescence reagent (Cyvita) and visualized. The strips were evaluated by light gray value analysis using ImageJ software (version 1.46; National Institutes of Health).

Reverse transcription-quantitative PCR (RT- $q P C R)$ analysis. Total RNA was extracted from OSCC cells using TRIzol ${ }^{\circledR}$ reagent (Invitrogen; Thermo Fisher Scientific, Inc.) according to the manufacturer's protocol. Total RNA was then reverse transcribed into cDNA using the HiScript ${ }^{\mathrm{TM}}$ II qRT SuperMix (Vazyme Biotech Co., Ltd.). Reverse transcription reaction condition was as following: $25^{\circ} \mathrm{C}$ For $5 \mathrm{~min}, 42^{\circ} \mathrm{C}$ for $60 \mathrm{~min}$ and $80^{\circ} \mathrm{C}$ for $2 \mathrm{~min}$. cDNA was analyzed by qPCR using the ChamQ ${ }^{\mathrm{TM}}$ Universal SYBR qPCR Master mix (Vazyme Biotech Co., Ltd.) according to the manufacturer's instructions. The amplification conditions were as follows: Pre-denaturation at $95^{\circ} \mathrm{C}$ for $10 \mathrm{~min}$; followed by 40 cycles of denaturation at $95^{\circ} \mathrm{C}$ for $10 \mathrm{sec}$, annealing at $60^{\circ} \mathrm{C}$ for $20 \mathrm{sec}$ and extension at $72^{\circ} \mathrm{C}$ for $34 \mathrm{sec}$. Primer sequences were listed as following: GAPDH, forward: 5'-CTTTGGTATCGTGGA AGGACTC-3' and reverse: 5'-GTAGAGGCAGGGATGATG TTCT-3'; Bcl-2, forward: 5'-GATCCTCGAGATGGCGCA CGCTGGGAGAAC-3' and reverse: 5'-GATCGGATCCTC ATGGCTGAGCGCAG-3'; Bax, forward: 5'-GGACGAACT GGACAGTAACATGG-3' and reverse: 5'-GCAAAGTAG AA-AAGGGCGACAAC-3'; p65, forward: 5'-ACAACAACC CCTTCCAAGAAGA-3' and reverse: 5'-CAGCCTGGTCCC GTGAAATA-3'; AKT, forward: 5'-TAAAGAAGGAGGTCA TCGTGG-3' and reverse: 5'-CGGGACAGGTGGAAGAAA A-3'. The relative gene expression levels were analyzed using 

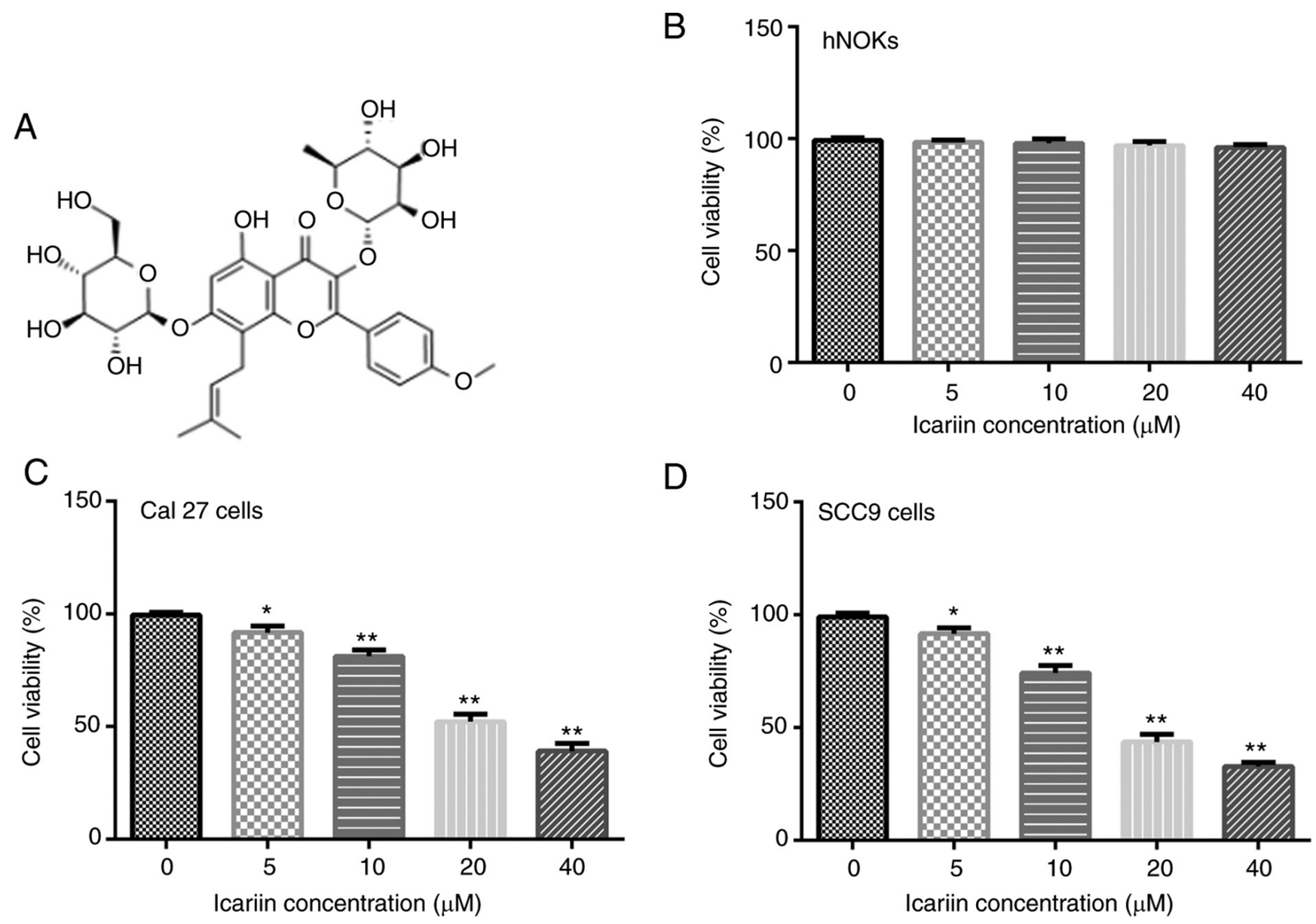

Figure 1. Effect of ICA on OSCC cell viability. (A) Chemical molecular structure of ICA. (B) hNOKs were treated with $0,5,10,20$ or $40 \mu \mathrm{M} \mathrm{ICA} \mathrm{for} 48 \mathrm{~h}$, and then a MTT assay was performed to measure cell viability. (C and D) OSCC cell lines, Cal 27 and SCC9, were treated with 0,5, 10, 20 or 40 $\mu \mathrm{M}$ ICA for $48 \mathrm{~h}$, and then a MTT assay was performed to measure cell viability. ${ }^{*} \mathrm{P}<0.05$ and ${ }^{* *} \mathrm{P}<0.01$ vs. $0 \mu \mathrm{M}$ ICA control group. ICA, icariin; OSCC, oral squamous cell carcinoma; hNOKs, human normal oral keratinocytes.

the $2^{-\Delta \Delta \mathrm{Cq}}$ method (25). All experiments were performed in triplicate.

Statistical analysis. The data from three independent experiments are expressed as the mean \pm standard deviation. Statistical analysis was carried out using GraphPad Prism 5 software (GraphPad Software, Inc.). The statistical differences among different groups were analyzed by one-way ANOVA followed by Tukey's post hoc tests. $\mathrm{P}<0.05$ was considered to indicate a statistically significant difference.

\section{Results}

Effect of ICA on the OSCC cell lines, Cal 27 and SCC9. The chemical molecular structure of ICA is shown in Fig. 1A. First, the effect of ICA on the proliferation of human normal oral keratinocytes (hNOKs) was determined, and the results indicated that there was no significant effect of ICA on the viability of hNOKs (Fig. 1B). Then, the effect of ICA on OSCC cells investigated and Cal 27 and SCC 9 cell lines were treated with $0,5,10,20$ or $40 \mu \mathrm{M}$ ICA for $48 \mathrm{~h}$. The effect of ICA on cell viability was determined using a MTT assay. The results demonstrated that ICA inhibited Cal 27 and SCC9 cell viability in a dose-dependent manner (Fig. 1C and D).
Effect of ICA on OSCC cell apoptosis and expression of apoptosis-related proteins. The results revealed that following treatment of Cal 27 cells with ICA $(0,5,10,20$ or $40 \mu \mathrm{M})$ for $48 \mathrm{~h}$, the apoptosis rate was significantly increased with increasing concentrations of ICA (Fig. 2A and B). Furthermore, the protein expression levels of cleaved-caspase-3 in Cal 27 cells were upregulated, those of pro-caspase- 3 were downregulated and the ratio of cleaved-caspase-3/pro-caspase-3 was increased, in a dose-dependent manner (Fig. 2C and D). Besides, it was demonstrated that the protein and mRNA expression levels of Bax (Fig. 2E and F) in Cal 27 cells were upregulated, and those of Bcl-2 (Fig. 2E and G) were downregulated in a dose-dependent manner. Additionally, the apoptosis rate results (Fig. 3A and B) and the expression levels of the apoptosis-related proteins (Fig. 3C-G) in SCC9 cells were consistent with those observed in the Cal 27 cell line.

Effect of ICA on the NF- $\kappa B$ and PI3K/AKT signaling pathways in OSCC cell lines. Cal 27 cells were treated with ICA (0, 5, 10,20 or $40 \mu \mathrm{M}$ ) for $48 \mathrm{~h}$, and the protein expression levels of p-p65, p65, p-AKT and AKT were detected using western blot analysis. The protein expression levels of p-p65 and p-AKT in Cal 27 cells were decreased with increasing concentrations of ICA (Fig. 4A). In addition, the p-p65/p65 and p-AKT/AKT 


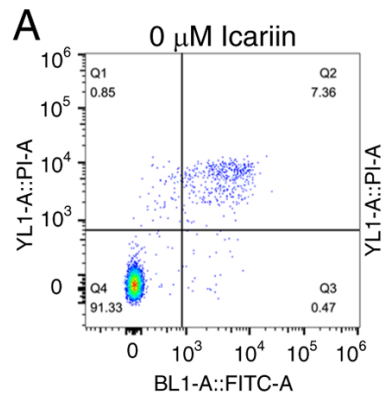

$\mathrm{B}$

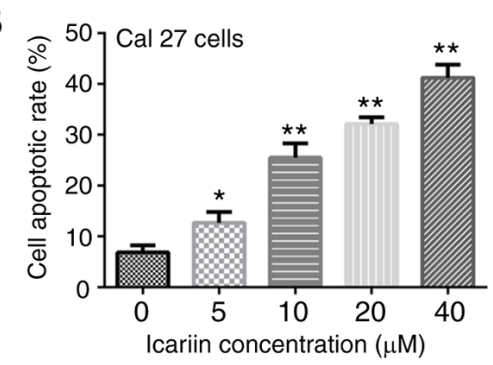

$E$

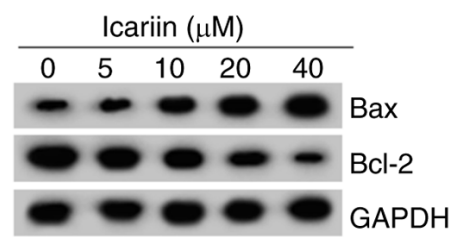

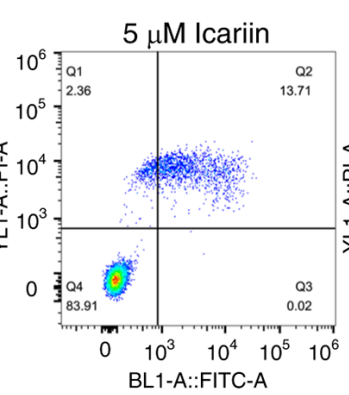

C
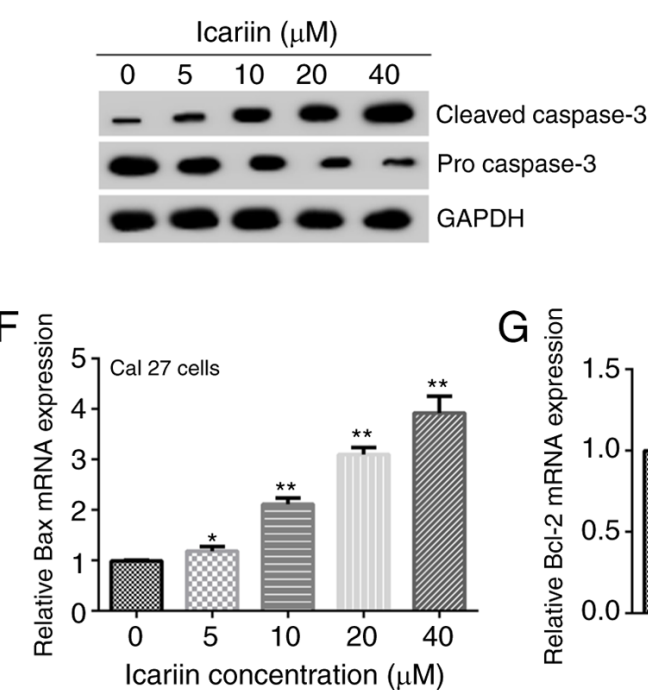
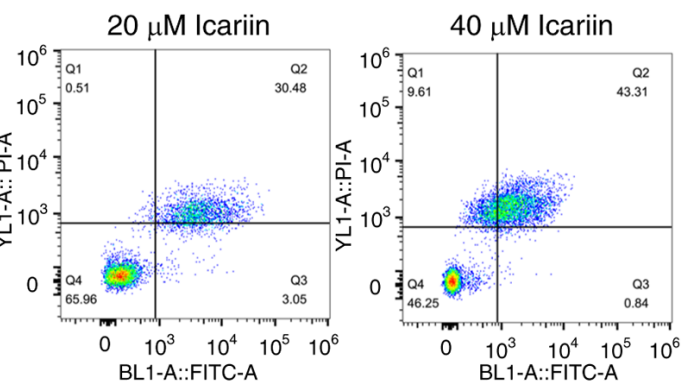

D

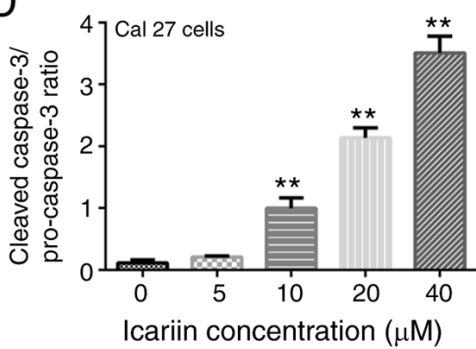

$G$.

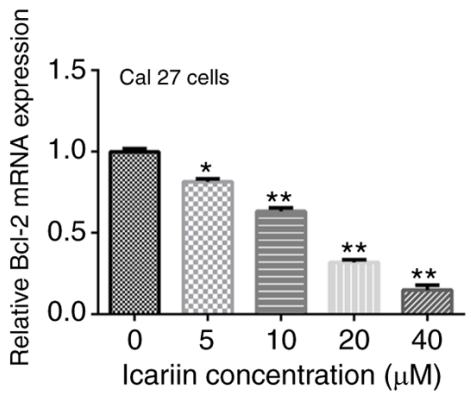

Figure 2. Apoptosis rate of the ICA-induced oral squamous cell carcinoma cell line, Cal 27. (A and B) Apoptosis rate of Cal 27 cells treated with $0,5,10,20$ or $40 \mu \mathrm{M}$ ICA for $48 \mathrm{~h}$ was determined by flow cytometric analysis. (C) Protein expression levels of cleaved-caspase-3 and pro-caspase-3 were detected using western blot analysis. (D) Cleaved-caspase-3/pro-caspase-3 ratio in Cal 27 cells is shown. (E) Protein expression levels of Bax and Bcl-2 were detected using western blot analysis. (F) mRNA expression levels of Bax were detected using RT-qPCR. (G) mRNA expression levels of Bcl-2 were detected using RT-qPCR. ${ }^{*} \mathrm{P}<0.05$ and ${ }^{* *} \mathrm{P}<0.01$ vs. $0 \mu \mathrm{M}$ ICA control group. ICA, icariin; RT-q, reverse transcription-quantitative.

protein ratios were reduced in Cal 27 cells treated with ICA (Fig. 4B and C). The RT-qPCR results revealed no significant changes in the mRNA expression levels of p65 and AKT (Fig. 4D and E). Similar results were obtained in SCC9 cells (Fig. 5). The aforementioned findings indicated that treatment of OSCC cells with ICA could attenuate cell proliferation and induce apoptosis via inhibiting the NF- $\kappa \mathrm{B}$ and PI3K/AKT signaling pathways.

\section{Discussion}

The present study aimed to investigate the effects of ICA in OSCC cells. The results showed that treatment with ICA significantly increased the apoptosis rate in a dose- and time-dependent manner. In OSCC cells treated with different concentrations of ICA, the protein expression of cleaved-caspase-3 and pro-caspase-3 were up- and downregulated, respectively, in a dose-dependent manner. Furthermore, ICA could attenuate OSCC cell proliferation via inhibiting the $\mathrm{NF}-\kappa \mathrm{B}$ and $\mathrm{PI} 3 \mathrm{~K} / \mathrm{AKT}$ signaling pathways. These findings provided novel insights into the role of ICA and potential for the management and clinical treatment of OSCC.

With increasing research in tumor biology and immunology, it has been gradually considered that the occurrence and development of malignant tumors depend not only on the tumor cells themselves, but also on their regulation by the immune system (26). Therefore, ICA can regulate the function of immune organs and cells, and enhance the activity of immune cytokines, thus improving tumor immune function (27). It has been reported that the $\mathrm{Bax} / \mathrm{Bcl}-2$ ratio is increased with increasing concentrations of ICA (3). In addition, a study demonstrated that ICA is involved in the human immune regulation via increasing the activity of $\mathrm{NF}-\kappa \mathrm{B}$ in anti-inflammatory- and antiviral-related pathways, inducing the expression of $\mathrm{p} 65$, accelerating lymphocyte apoptosis and delaying immune aging (28). Furthermore, ICA could inhibit the proliferation of acute promyelocytic leukemia cells in a dose-dependent manner, thereby promoting their apoptosis (4). Additionally, a study revealed that ICA could block the $N F-\kappa B$ signaling pathway, which in turn could hinder the mRNA expression of the inflammation-related factor inducible nitric oxide synthase and delay the onset of inflammatory diseases, thus suggesting that ICA has strong anti-inflammatory effects (2). Therefore, it was hypothesized that ICA could also inhibit the NF- $\mathrm{NB}$ and $\mathrm{PI} 3 \mathrm{~K} / \mathrm{AKT}$ signaling pathways in OSCC, thereby attenuating OSCC cell proliferation and inducing apoptosis.

In the current study, the effect of ICA on Cal 27 and SCC9 cell viability and apoptosis was assessed by MTT and 


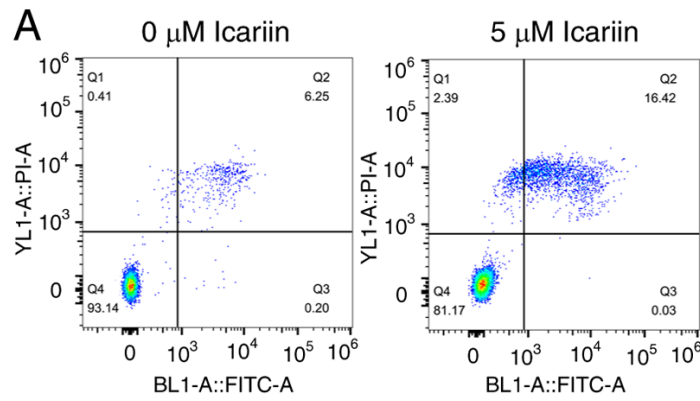

B

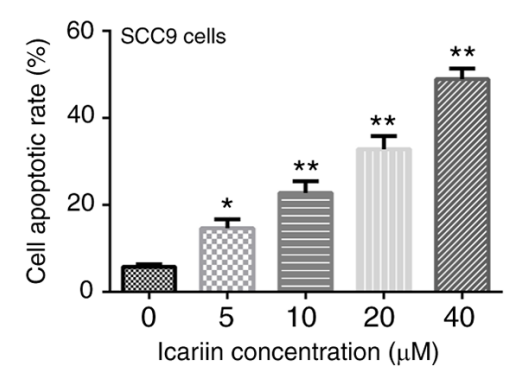

$E$

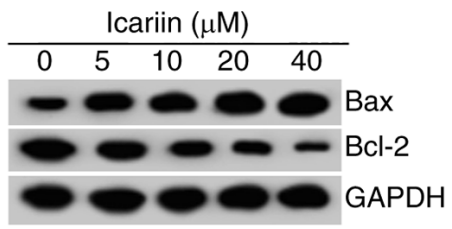

C

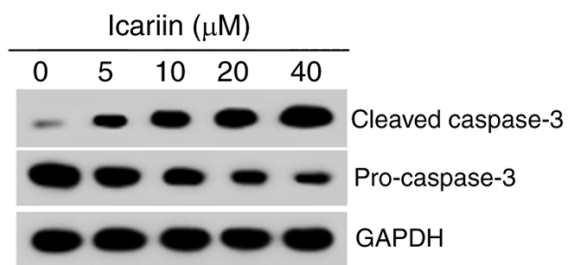

$20 \mu \mathrm{M}$ Icariin

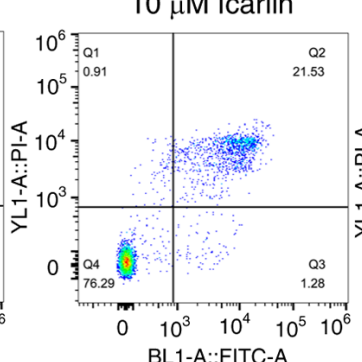

BL1-A::FITC-A
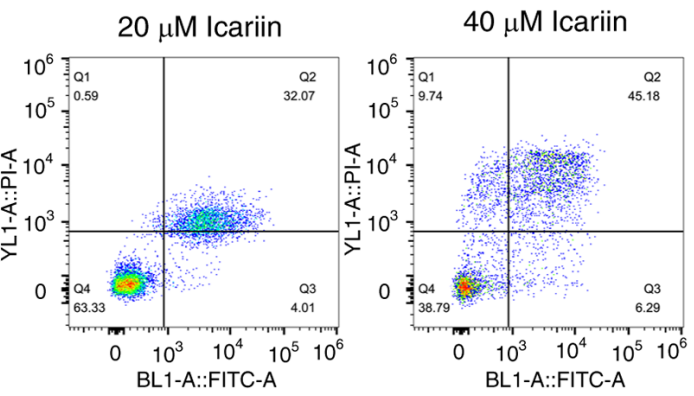

$\mathrm{D}$

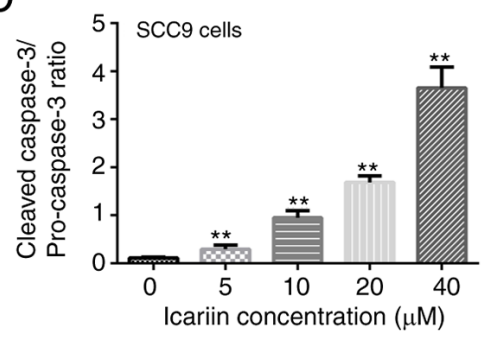

$F \subsetneq$

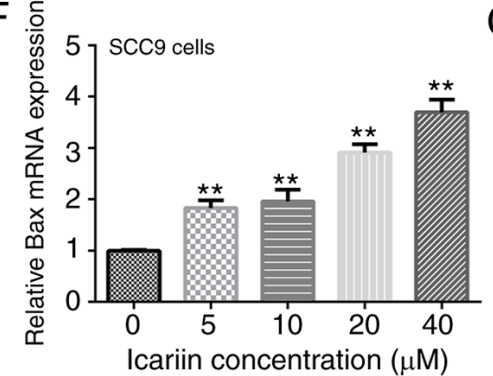

$\mathrm{G}$

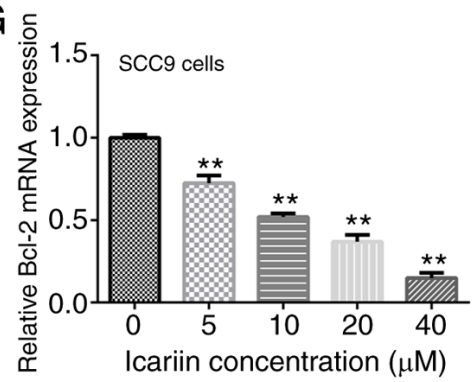

Figure 3. Apoptosis rate of the ICA-induced oral squamous cell carcinoma cell line, SCC9. SCC9 cells were treated with $0,5,10,20$ or $40 \mu \mathrm{M}$ ICA for $48 \mathrm{~h}$. (A and B) Apoptosis rate of SCC9 cells treated with 0, 5, 10, 20 or $40 \mu \mathrm{M}$ ICA for $48 \mathrm{~h}$ was determined using flow cytometric analysis. (C) Protein expression levels of cleaved-caspase-3 and pro-caspase-3 were detected using western blot analysis. (D) Cleaved-caspase-3/pro-caspase-3 ratio in SCC9 cells is shown. (E) Protein expression levels of Bax and Bcl-2 were detected using western blot analysis. (F) mRNA expression levels of Bax were detected using RT-qPCR. (G) mRNA expression levels of Bcl-2 were detected using RT-qPCR. " $\mathrm{P}<0.05$ and ${ }^{* *} \mathrm{P}<0.01$ vs. $0 \mu \mathrm{M}$ ICA control group. ICA, icariin; RT-q, reverse transcription-quantitative.
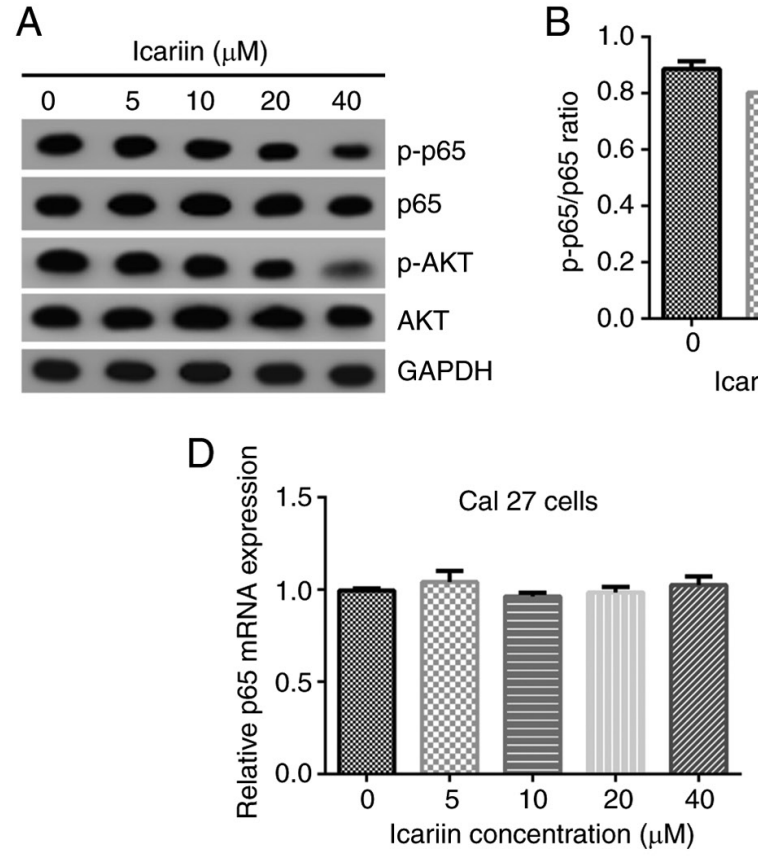
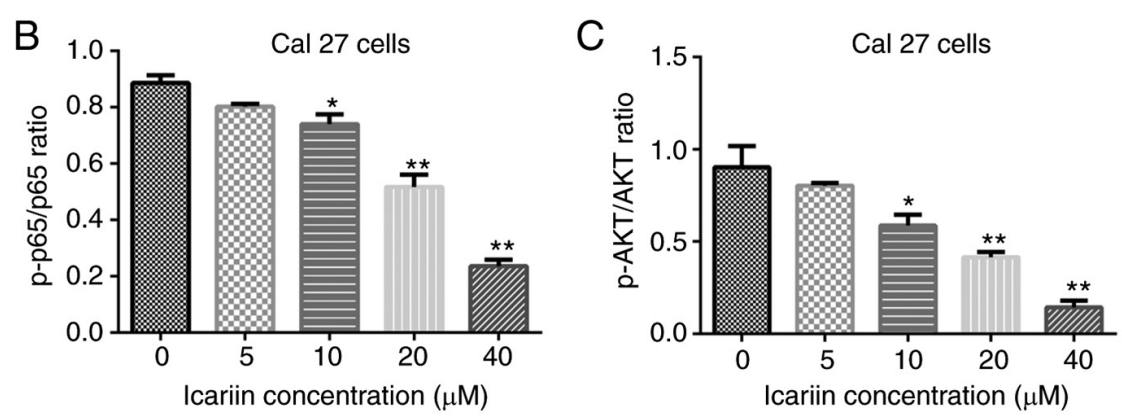

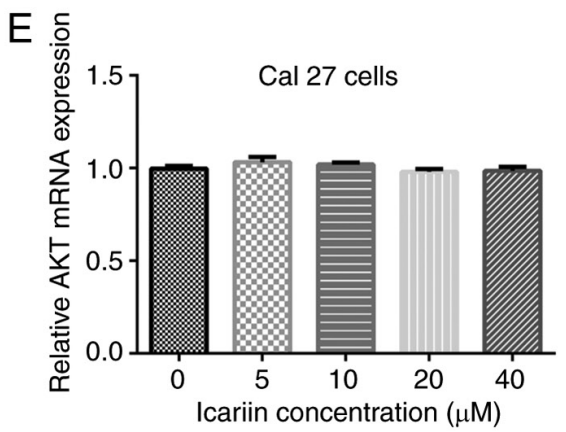

Figure 4. Effect of ICA on the of nuclear factor- $\mathrm{KB}$ and phosphatidylinositol-3-kinase/AKT signaling pathways in Cal 27 cells. (A) Protein expression levels of p-p65, p65, p-AKT and AKT were determined using western blot analysis. (B) p-p65/p65 ratio. (C) p-AKT/AKT. (D) mRNA expression levels of p65. (E) mRNA expression levels of AKT. ${ }^{*} \mathrm{P}<0.05,{ }^{* *} \mathrm{P}<0.01$ vs. $0 \mu \mathrm{M}$ ICA control group. ICA, icariin; p-, phosphorylated; AKT, protein kinase B. 
A

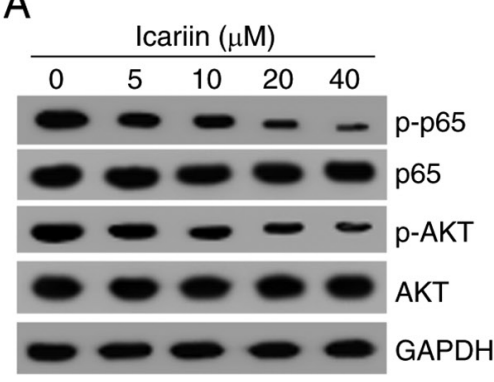

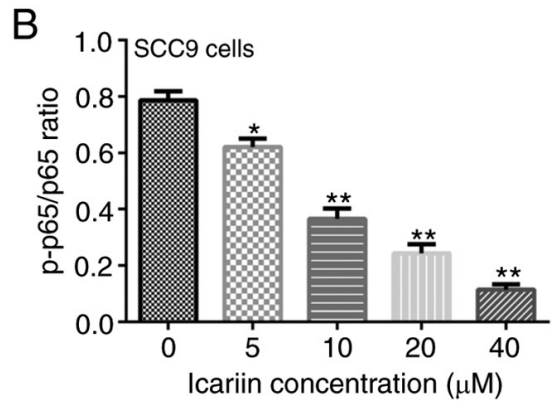

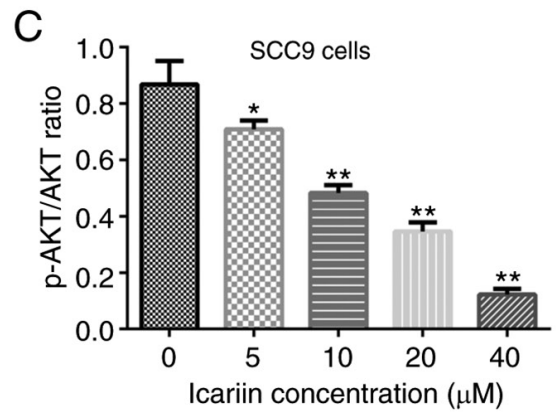

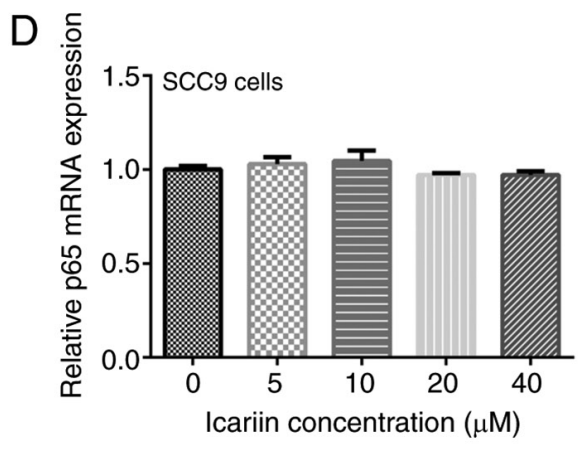

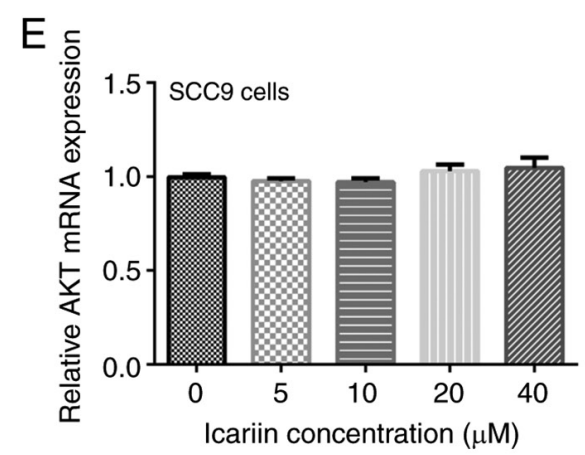

Figure 5. Effect of ICA on the nuclear factor- $\mathrm{B}$ and phosphatidylinositol-3-kinase/AKT signaling pathways in SCC9 cells. (A) Protein expression levels of p-p65, p65, p-AKT and AKT were determined using western blot analysis. (B) p-p65/p65 ratio. (C) p-AKT/AKT ratio. (D) mRNA expression levels of p65. (E) mRNA expression levels of AKT. ${ }^{*} \mathrm{P}<0.05$ and ${ }^{* *} \mathrm{P}<0.01$, compared with the $0 \mu \mathrm{M}$ ICA control group. ICA, icariin; p-, phosphorylated; AKT, protein kinase B.

western blot assays, respectively. The results revealed that ICA could attenuate cell viability and induce apoptosis in the OSCC cell lines in a dose-dependent manner. Chen et al (2) demonstrated that ICA could upregulate the protein expres-

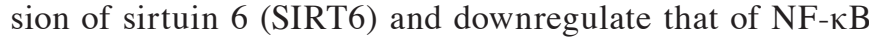
(p65) in animal tissues and cell models. These results showed that the ICA-mediated SIRT6 upregulation had an inhibitory effect on the NF- $\kappa \mathrm{B}$ inflammatory signaling pathway, since treatment with ICA decreased the mRNA expression levels of the NF- $\kappa B$ downstream target genes, TNF- $\alpha$, intercellular adhesion molecule 1, IL-2 and IL-6. Herein, the protein expression levels of p-p65 and p-AKT were decreased, following cell treatment with ICA, in a dose-dependent manner. No obvious changes were observed regarding the mRNA expression levels of p65 and AKT in both OSCC cell lines. In addition, the p-p65/p65 and p-AKT/AKT ratios were decreased in OSCC cells in a dose-dependent manner. The aforementioned findings suggested that ICA could attenuate OSCC cell proliferation via inhibiting the NF- $\kappa B$ and PI3K/AKT signaling pathways.

ICA is a novel biological immunomodulator and inducer of differentiation, thus improving immunity function, and inhibiting tumor cell proliferation, tumor growth and tumor angiogenesis $(27,29,30)$. Herein, ICA inhibited the NF- $\mathrm{KB}$ and PI3K/AKT signaling pathways to promote OSCC cell proliferation, suggesting that this drug should be considered as a potential monomer for treating OSCC. However, the current study still had some limitations. For example, the effect of ICA was only studied in two OSCC cell lines, and so more cell lines need to be used for verification. Furthermore, the effects of only four concentrations of ICA on OSCC cell lines were studied, thus more concentrations of ICA should be explored.
In addition, no in vivo studies were conducted. In the future, these issues will be studied in more depth.

\section{Acknowledgements}

Not applicable.

\section{Funding}

No funding was received.

\section{Availability of data and materials}

The datasets used and/or analyzed during the current study are available from the corresponding author on reasonable request.

\section{Authors' contributions}

LS contributed to study design, data collection, statistical analysis, data interpretation and manuscript preparation. JZ contributed to data collection, statistical analysis and manuscript preparation. All authors read and approved the final manuscript. LS and JZ confirm the authenticity of all the raw data.

\section{Ethics approval and consent to participate}

Not applicable.

\section{Patient consent for publication}

Not applicable. 


\section{Competing interests}

The authors declare that they have no competing interests.

\section{References}

1. Qin L, Zhang G, Sheng H, Wang XL, Wang YX, Yeung KW, Griffith JF, Li ZR, Leung KS and Yao XS: Phytoestrogenic compounds for prevention of steroid-associated osteonecrosis. J Musculoskelet Neuronal Interact 8: 18-21, 2008.

2. Chen Y, Sun T, Wu J, Kalionis B, Zhang C, Yuan D, Huang J, Cai W, Fang $\mathrm{H}$ and Xia S: Icariin intervenes in cardiac inflammaging through upregulation of SIRT6 enzyme activity and inhibition of the NF-kappa B pathway. Biomed Res Int 2015: 895976, 2015.

3. Carlsten M and Childs RW: Genetic manipulation of NK cells for cancer immunotherapy: Techniques and clinical implications. Front Inmunol 6: 266, 2015.

4. Wang Z, Zhang H, Dai L, Song T, Li P, Liu Y and Wang L: Arsenic trioxide and icariin show synergistic anti-leukemic activity. Cell Biochem Biophys 73: 213-219, 2015.

5. Huang S, Xie T and Liu W: Icariin inhibits the growth of human cervical cancer cells by inducing apoptosis and autophagy by targeting mTOR/PI3K/AKT signalling pathway. J BUON 24 : 990-996, 2019.

6. Wang P, Zhang J, Xiong X, Yuan W, Qin S, Cao W, Dai L, Xie F, Li A and Liu Z: Icariin suppresses cell cycle transition and cell migration in ovarian cancer cells. Oncol Rep 41: 2321-2328, 2019.

7. Kim B, Seo JH, Lee KY and Park B: Icariin sensitizes human colon cancer cells to TRAIL-induced apoptosis via ERK-mediated upregulation of death receptors. Int J Oncol 56: $821-834,2020$

8. Song L, Chen X, Mi L, Liu C, Zhu S, Yang T, Luo X, Zhang Q, $\mathrm{Lu} \mathrm{H}$ and Liang X: Icariin-induced inhibition of SIRT6/NF- $\kappa \mathrm{B}$ triggers redox mediated apoptosis and enhances anti-tumor immunity in triple-negative breast cancer. Cancer Sci 111: 4242-4256, 2020.

9. Ju ZY, Tang QM, Chen LL, et al: Axl inhibitor R428 induces autophagy of oral squamous cell carcinoma Cal27 cells. J Pract Stomatol 35: 2019.

10. Hu XG, Qiu ZL, Zen JC, et al: Differential expression of microRNAs in human oral squamous cell carcinoma stem cells. J Pract Stomatol 3: 398-402, 2019.

11. Schneider P, Schon M and Pletz N, Seitz CS, Liu N, Ziegelbauer K Zachmann K, Emmert S and Schön MP: The novel PI3 kinase inhibitor, BAY80-6946, impairs melanoma growth in vivo and in vitro. Exp Dermatol 23: 579-584, 2014

12. Liu YJ, Huang LL, Hao BH, Li H, Zhu S, Wang Q, Li R, Xu Y and Zhang X: Use of an osteoblast overload damage model to probe the effect of icariin on the proliferation, differentiation and mineralization of MC3T3-E1cells through the Wnt/ $\beta$-catenin signalling pathway. Cell Physiol Biochem 41: 1605-1615, 2017.

13. Li W, Wang M, Wang LY, Ji S, Zhang J and Zhang C: Icariin synergizes with arsenic trioxide to suppress human hepatocellular carcinoma. Cell Biochem Biophys 68: 427-436, 2014.

14. Yang LJ, Wang YX, Guo H and Guo M: Synergistic anti-cancer effects of icariin and temozolomide in glioblastoma. Cell Biochem Biophys 71: 1379-1385, 2015.

15. Shi DB, Li XX, Zheng HT, Li DW, Cai GX, Peng JJ, Gu WL, Guan ZQ, Xu Y and Cai SJ: Icariin-mediated inhibition of NF-кB activity enhances the in vitro and in vivo antitu-mour effect of 5-fluorouracil in colorectal cancer. Cell Biochem Biophys 69: $523-530,2014$
16. Xu W, Tao $\mathrm{H}$ and Zhang Q: Effect of Six1 gene on $\mathrm{As}_{2} \mathrm{O}_{3}$-induced oral squamous cell carcinoma cell apoptosis and ROS levels. J Clin Exp Medicine 2019; (15).

17. Peng $M$ and Pang C: MicroRNA-140-5p inhibits the tumorigenesis of oral squamous cell carcinoma by targeting p21-activated kinase 4. Cell Biol Int: Aug 8, 2019 (Epub ahead of print).

18. Wang XM, Liu CM, Zhang CR, Xu XG and Fu SB: Functional significance of TGF-betal signal transduction pathway in oral squamous cell carcinoma. Zhonghua Zhong Liu Za Zhi 31: 28-32, 2009 (In Chinese).

19. Chen Z, He J, Xing X, Li P, Zhang W, Tong Z, Jing X, Li L, Liu D, Wu Q and Ju H: Mn12Ac inhibits the migration, invasion and epithelial-mesenchymal transition of lung cancer cells by downregulating the Wnt/ $\beta$-catenin and PI3K/AKT signaling pathways. Oncol Lett 16: 3943-3948, 2018.

20. Xie J, Lin W, Huang L, Xu N, Xu A, Chen B, Watanabe M, Liu C and Huang P: Bufalin suppresses the proliferation and metastasis of renal cell carcinoma by inhibiting the PI3K/Akt/mTOR signaling pathway. Oncol Lett 16: 3867-3873, 2018

21. Choi MS, Moon SM, Lee SA, Park BR, Kim JS, Kim DK, Kim YH and Kim CS: Adenosine induces intrinsic apoptosis via the PI3K/Akt/mTOR signaling pathway in human pharyngeal squamous carcinoma FaDu cells. Oncol Lett 15: 6489-6496, 2018.

22. Hao Y, Zhang C, Sun Y and Xu H: Licochalcone A inhibits cell proliferation, migration, and invasion through regulating the PI3K/AKT signaling pathway in oral squamous cell carcinoma. Onco Targets Ther 12: 4427-4435, 2019.

23. Fan QC, Tian H, Wang Y and Liu XB: Integrin-alpha5 promoted the progression of oral squamous cell carcinoma and modulated PI3K/AKT signaling pathway. Arch Oral Biol 101: 85-91, 2019.

24. Velu P, Vijayalakshmi A and Vinothkumar V: Inhibiting the PI3K/Akt, NF-kB signalling pathways with syringic acid for attenuating the development of oral squamous cell carcinoma cells SCC131. J Pharm Pharmacol 72: 1595-1606, 2020.

25. Ferlay J, Soerjomataram I, Dikshit R, Eser S, Mathers C, Rebelo M, Parkin DM, Forman D and Bray F: Cancer incidence and mortality worldwide: Sources, methods and major patterns in GLOBOCAN 2012. Int J Cancer 136: E359-E386, 2015.

26. Galluzzi L and Rudqvist NP: Preface: More than two decades of modern tumor immunology. Methods Enzymol 629: xxii-xlii, 2019.

27. Zhang X, Kang Z, Li Q, Zhang J, Cheng S, Chang H, Wang S, Cao S, Li T, Li J, et al: Antigen-adjuvant effects of icariin in enhancing tumor-specific immunity in mastocytoma-bearing DBA/2J mice. Biomed Pharmacother 99: 810-816, 2018.

28. Gao M and Li W: In vitro study on the inhibitory effect of icariin on human bladder cancer T24 cells. Shijiazhuang: Hebei Medical University 2010.

29. Hao H, Zhang Q, Zhu H, Wen Y, Qiu D, Xiong J, Fu X, Wu Y, Meng $\mathrm{K}$ and $\mathrm{Li} \mathrm{J}$ : Icaritin promotes tumor T-cell infiltration and induces antitumor immunity in mice. Eur J Immunol 49: 2235-2244, 2019

30. Tan HL, Chan KG, Pusparajah P, Saokaew S, Duangjai A, Lee LH and Goh BH: Anti-cancer properties of the naturally occurring aphrodisiacs: Icariin and its derivatives. Front Pharmacol 7: 191, 2016.

This work is licensed under a Creative Commons Attribution-NonCommercial-NoDerivatives 4.0 International (CC BY-NC-ND 4.0) License. 